\title{
Estimation de la qualité bactériologique du lait cru par microrespirométrie
}

\author{
D Rongvaux-Gaïda ${ }^{1 *}$, A Peroz ${ }^{1}$, B Verdier ${ }^{1}$, C Piton ${ }^{2}$ \\ 1 CNRS UA 689 laboratoire d'écologie générale, Museum National d'Histoire Naturelle, 4 avenue \\ du Petit-Château, 91800 Brunoy; \\ ${ }^{2}$ INRA, station de recherches en technologie et analyses laitières, $B P n^{\circ} 89$, place du-Champ- \\ de-Foire, 39800 Poligny, France
}

(Reçu le 16 février 1989; accepté le 18 septembre 1989)

\begin{abstract}
Résumé - Les caractéristiques de la technique microrespirométrique à pression et volume variables (sensibilité et possibilité d'automatisation) ont permis d'envisager son utilisation pour l'évaluation de la qualité bactériologique du lait cru. La première partie du travail a consisté à établir les conditions de mesure permettant l'estimation de la consommation d'oxygène par les bactéries présentes dans le lait cru. En effet, lors d'une incubation d'un échantillon à $30^{\circ} \mathrm{C}$, la consommation initiale du lait est due essentiellement à l'activité respiratoire des cellules somatiques; au cours du temps, cette activité décroît pour devenir négligeable par rapport à celle des bactéries en phase de croissance exponentielle. Pour un volume de $100 \mu \mathrm{l}$, ces conditions de mesure sont atteintes, pour la plupart des échantillons, après une incubation de $4 \mathrm{~h}$ à $30^{\circ} \mathrm{C}$. II s'est alors avéré qu'il existait une relation linéaire entre les valeurs logarithmiques des consommations d'oxygène $\left(\mathrm{O}_{2(\mathrm{t})}\right)$ et des nombres d'UFC $/ \mathrm{ml}$ mesurés simultanément après une incubation de $4 \mathrm{~h}$ minimum à $30^{\circ} \mathrm{C}$. Le coefficient de corrélation obtenu sur 43 échantillons de lait cru, couvrant une gamme de concentration de $5 \times 10^{4}$ à $5 \times 10^{7} \mathrm{UFC} / \mathrm{ml}$, était de 0,958 et l'écart type résiduel de $0,225 \log _{10} \mathrm{UFC} / \mathrm{ml}$. Ces résultats ont permis d'envisager une prédiction de la consommation bactérienne initiale $\left(\mathrm{O}_{2(t 0)}\right)$ à partir des valeurs mesurées en phase exponentielle. Ainsi, dans la deuxième partie de ce travail, 94 échantillons de lait couvrant une gamme de concentration de $6 \times 10^{3}$ à $10^{7} \mathrm{UFC} / \mathrm{ml}$ ont été analysés en double par la technique de référence et par la microrespirométrie. Le coefficient de corrélation obtenu entre les valeurs logarithmiques des consommations d'oxygène bactériennes initiales prédites $\left(\mathrm{O}_{2(\mathrm{to})}\right)$ et des nombres d'UFC/ml était de 0,973 et l'écart type de $0,177 \log _{10} \mathrm{UFC} / \mathrm{ml}$.
\end{abstract}

lait cru / dénombrement microbien / microrespirométrie / consommation d'oxygène / justesse

Summary - Estimation of the bacteriological quality of raw milk by microrespirometry. A new microrespirometer, based upon the measurement of consumed oxygen at variable pressure and volume, was used to assess the bacteriological quality of raw milk. In the first part of this work, the measurement conditions allowing the estimation of oxygen consumption by bacteria present in raw milk, were developed. During incubation at $30^{\circ} \mathrm{C}$, the initial $\mathrm{O}_{2}$ consumption of a milk sample is essentially due to the activity of somatic cells; this activity decreases and becomes insignificant com-

\footnotetext{
* Adresse actuelle : INRA, station de recherches en technologie et analyses laitières, BP 89, 39800 Poligny, France.
} 
pared to the bacterial activity during the exponential growth phase. With a test sample volume of 100 $\mu l$, the measuring conditions are fulfilled for the most part of samples after an incubation period of $4 \mathrm{~h}$ at $30^{\circ} \mathrm{C}$. It has been demonstrated that there is a linear relationship between the $\log _{10}$ number of bacteria and the $\log _{10}$ amount of oxygen consumed $\left(\mathrm{O}_{2(t)}\right)$ measured simultaneously after a $4 \mathrm{~h}$ incubation period. The coefficient of correlation obtained on 43 raw milk samples, ranging from $5 \times 10^{4}$ to $5 \times 10^{7}$ CFU/ml was 0.958 , with a residual standard deviation of $0.225 \log _{10}$ CFU/ml. These results allow us to establish a method of predicting the bacterial initial $\mathrm{O}_{2}$ consumption $\left(\mathrm{O}_{2(t o)}\right)$ from the values obtained during the exponential phase. Therefore, in the second part of this work, 94 samples ranging from $6 x$ $10^{3}$ to $10^{7} \mathrm{CFU} / \mathrm{ml}$ were analysed in duplicate by the reference method and by microrespirometry. The correlation coefficient obtained between the $\log _{10}$ number of bacteria and the $\log _{10}$ predicted initial oxygen consumption $\left(\mathrm{O}_{2(\mathrm{t})}\right)$ was 0.973 with a residual standard deviation of $0.177 \log _{10} \mathrm{CFU} / \mathrm{ml}$.

raw milk / microrespirometry / oxygen consumption / colony counts / accuracy

\section{INTRODUCTION}

Les méthodes normalisées de numération de la flore totale du lait cru sont basées sur le dénombrement des colonies formées dans un milieu nutritif gélosé non sélectif et présentent un délai de réponse de $72 \mathrm{~h}$, incompatible avec les besoins de l'industrie laitière. Au cours des dernières années, les exigences de rapidité et/ou de réduction de coût au sein des entreprises, ont entraîné le développement de nombreuses techniques indirectes basées sur la mesure d'un signal significatif d'un certain niveau et de l'activité des microorganismes dans le lait.

Après l'introduction de la réfrigération du lait à la ferme, les tests de réduction des colorants (bleu de méthylène, résazurine) ont été abandonnés car ils ne présentaient plus qu'une faible corrélation avec les techniques de référence (Luck, 1972) notamment en raison du rôle très important de la nature de la flore microbienne sur la chute du potentiel redox du lait. Une mesure de consommation d'oxygène est alors apparue comme une voie intéressante étant donné que toutes les bactéries aérobies utilisent l'oxygène comme accepteur final des électrons dans la chaîne respiratoire. Les premiers tests réalisés consistaient en la mesure d'une diminution du taux $d^{\prime} \mathrm{O}_{2}$ dissous dans l'échantillon à l'aide d'électrodes spécifiques. Cependant, ils n'ont pas abouti aux résultats escomptés, même après standardisation de la méthode par saturation des échantillons en oxygène, car celle-ci ne prend pas en compte le flux $\mathrm{d}^{\prime} \mathrm{O}_{2}$ qui est en fait le seul paramètre directement lié à l'augmentation de la population bactérienne (Pickering et Jayne-Williams, 1963; Stadhouders, 1965; Hadland, 1966; Luck et al, 1968 et $1970 \mathrm{a})$.

L'UA 689 du CNRS a mis au point un microrespiromètre à pression et volume variables ou manovolumétrique (Verdier, 1983) qui permet de suivre la dynamique des gaz propre à de faibles quantités de substrats biologiques, comme par exemple des consommations d'oxygène par respiration ou des productions de $\mathrm{CO}_{2}$ par fermentation. Le fondement de toute technique gazométrique est la loi d'Etat des gaz : $N R T=P \cdot V$, mais le principe original du microrespiromètre manovolumétrique repose sur l'exploitation de l'intégrité de la forme différentielle de cette loi $: \mathrm{d} R N T=P d V+$ $V \cdot \mathrm{d} P$. La dépendance des paramètres d'état, pression et volume, permet d'associer la simplicité d'utilisation des systèmes à pression constante (type Warburg) à la sensibilité élevée des systèmes à volume constant (Cartesian divers). Les perfor- 
mances réalisées par cet appareil sur des milieux biologiques variés, sa sensibilité, ses possibilités d'automatisation et de travail en série nous ont permis d'envisager son application pour l'estimation de la qualité bactériologique du lait cru.

Dans la première partie de ce travail, nous nous sommes proposés de définir, sur un prototype de laboratoire, les conditions expérimentales optimales pour cette application et de vérifier la validité d'une mesure de consommation d'oxygène pour estimer le niveau de pollution bactérienne du lait. En effet, la cinétique de consommation d'oxygène d'un échantillon de lait au cours du temps peut être décomposée en deux phases : un plateau et une phase de consommation exponentielle, tout à fait caractéristique de celle d'une croissance bactérienne. Cependant, le plateau ne peut être totalement attribué à une phase de latence de la flore microbienne du lait pour deux raisons principales : d'une part, sa durée n'est pas compatible avec les temps de latence généralement mesurés à une température de $30^{\circ} \mathrm{C}$ (moins de 10 min pour les bactéries mésophiles et psychrotrophes; Anonyme, 1980); d'autre part, les cellules somatiques présentes en quantité non négligeable dans le lait, ont une activité respiratoire qui varie selon l'origine, la durée et les conditions de conservation des échantillons (Luck et al, 1970b). La première partie de l'étude a donc consisté à déterminer la contribution des cellules somatiques à cette activité respiratoire initiale du lait, après avoir vérifié sur un lait stérile, l'absence de réactions oxydatives mesurables. Ensuite, nous avons étudié la relation existant entre une consommation d'oxygène mesurée au cours de la phase de croissance exponentielle et le niveau de la flore totale du lait au même instant. L'étroitesse de cette relation nous a permis d'envisager l'utilisation de la consommation d'oxygène bactérienne initiale, prédite à partir des valeurs mesurées en phase de croissance exponentielle, pour estimer le niveau de la flore totale dans l'échantillon de lait non incubé. Dans la seconde partie du travail, nous avons établi la relation entre le niveau initial de la flore totale du lait et la consommation d'oxygène bactérienne initiale prédite. Nous nous sommes limités à l'étude de la justesse en considérant que la fidélité ne peut être établie que sur une version plus élaborée de l'appareil.

\section{MATÉRIEL ET MÉTHODES}

\section{Origine des échantillons}

Cent trente-sept échantillons de lait cru, constitués du mélange de 2 traites ont été prélevés en tank de refroidissement. Parmi ces échantillons, 90 provenaient d'une ferme de la région de Brunoy et $\mathbf{4 7}$ de différents producteurs de la région de Poligny. Ces derniers échantillons étaient envoyés le soir par la poste et conservés à $0^{\circ} \mathrm{C}$ dans de la glace pilée durant le transport. Les échantillons étaient analysés dès leur arrivée au laboratoire, ou placés à une température comprise entre 5 et $10^{\circ} \mathrm{C}$ pendant 24 à $48 \mathrm{~h}$ pour obtenir des niveaux de contamination plus élevés.

A diverses reprises, deux échantillons de lait riche en cellules somatiques ont été prélevés de façon aseptique en tube stérile $(10 \mathrm{ml})$, soit dans un pot trayeur immédiatement après la traite, soit directement à la mamelle : lait $2^{\theta}$ jet. En effet, nous avons considéré qu'après élimination du premier jet souvent très chargé en bactéries, le lait prélevé aseptiquement à la sortie de la mamelle contenait moins de 5000 micro-organismes/ml (Richard et Auclair, 1967). La cinétique de consommation d'oxygène de ces échantillons a été suivie environ $30 \mathrm{~min}$ après la traite.

\section{Mode d'agitation des échantillons}

Avant chaque analyse, les échantillons ont été soumis à une agitation standard, consistant en 
25 mouvements aller-retour, d'une amplitude de $25-30 \mathrm{~cm}$, effectués en 7 à $10 \mathrm{~s}$ (Anonyme, 1972).

\section{Numération bactérienne}

Les échantillons ont été soumis au dénombrement des bactéries aérobies mésophiles sur milieu Plate Count Agar (Difco) après 3 jours d'incubation à $30^{\circ} \mathrm{C}$. Dans la première partie de l'étude, nous avons utilisé la méthode classique de dénombrement : dilutions décimales et incorporation de l'inoculum dans la gélose selon la norme provisoire FIL 100 (Anonyme, 1981). Dans la seconde partie de l'étude, le lait a été ensemencé à la surface de la gélose à l'aide de l'ensemenceur Spiral (Gilchrist et al, 1973), après dilution des échantillons dans une solution de bacto-peptone (Difco) à la concentration de $0,1 \%$. On admettra que le changement de technique d'ensemencement n'affecte pas les conclusions de l'étude car il a été largement montré que la méthode d'ensemencement en surface donne des résultats similaires à la technique classique de dénombrement (Campbell et Gilchrist, 1973; Gilchrist et al, 1973; Donnelly et al, 1976; Jarvis et al, 1977; Peeler et al, 1977).

Pour chaque échantillon, les analyses ont été effectuées en double afin d'obtenir une plus grande précision sur les résultats de dénombrement. Les résultats sont exprimés en unités formant colonies par millilitre de lait (UFC/ml).

\section{Mesure de la consommation d'oxygène}

\section{Description du microrespiromètre}

Le respiromètre prototype utilisé est constitué d'un caisson régulé en température au $1 / 100$ de degré et en pression à $0,2 \mathrm{~mm}$ d'eau, dans lequel peuvent être immergées 16 unités respirométriques. Une unité respirométrique (fig $1 \mathrm{~A}$ ) se compose d'une chambre d'incubation (Ch) surmontée d'un tube capillaire calibré (M) de 0,2 $\mathrm{mm}$ de diamètre (d) et de $350 \mathrm{~mm}$ de longueur, associé à un réservoir $(R)$ permettant le dépôt du liquide manovolumétrique. Un capteur de $\mathrm{CO}_{2}$ (C), constitué d'une pastille de fibre de verre imprégnée d'une solution de potasse est placé à la base du capillaire; cela permet d'affirmer que toute diminution de la quantité gazeuse correspond à une consommation d'oxygène, les paramètres température et pression étant maintenus constants.

\section{Utilisation du microrespiromètre}

L'échantillon de lait, préalablement agité, est disposé au fond de l'unité respirométrique à rai-
A

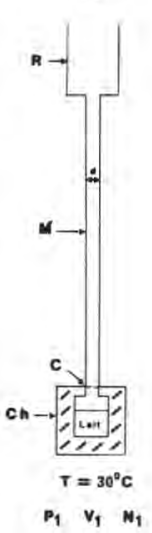

B

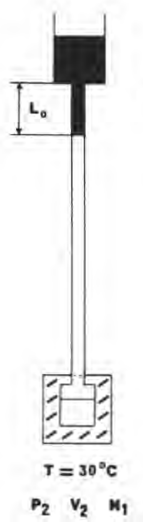

C

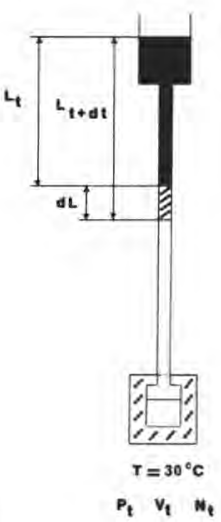

Fig 1. Représentation schématique d'une unité microrespirométrique à pression et volume variables (d'après Verdier, 1983): A - unité en attente; B - unité chargée; C-mesure.

$T=$ température; $P=$ pression; $V=$ volume; $N=$ nombre de moles; $R$ = réservoir; $M=$ capillaire; $d=$ diamètre du capillaire; $\mathrm{C}=$ capteur de $\mathrm{CO}_{2}$; $\mathrm{Ch}=$ chambre respirométrique; $L_{o}=$ longueur initiale d'équilibre de la colonne de liquide; $L_{t}=$ longueur de la colonne de liquide à l'instant $(t) ; \mathrm{d} t=$ espace de temps écoulé entre 2 mesures; $\mathrm{d} l=\mathrm{al}$ longement de la colonne de liquide entre 2 mesures.

Diagram of a microrespirometric unit at variable pressure and volume (according to Verdier, 1983): $A$ - unit before loading; $B$ - loaded unit; $C$ - measurement.

$\mathrm{T}=$ temperature $; \mathrm{P}=$ pressure $; \mathrm{V}=$ volume $; \mathrm{N}=$ number of moles; $R=$ reservoir; $M=$ glass capillary tube; $\mathrm{d}=$ capillary tube diameter; $\mathrm{C}=\mathrm{CO}_{2}$ fixator; $C h=$ respirometer cell; $L_{0}=$ initial length of balance of the fluid column; $L_{t}=$ length of the column at moment $(\mathrm{t}) ; \mathrm{dt}=$ time elapsed between 2 measurements; $\mathrm{dl}=$ elongation of the fluid column between 2 measurements. 
son de $100 \mu \mathrm{l}$ par chambre. Le dépôt de volumes plus importants aurait l'avantage d'augmenter la vitesse de réponse et la précision de lecture, mais il nécessite une agitation magnétique au sein de la chambre de façon à assurer une diffusion optimale des gaz au travers de l'échantillon de lait. Le prototype de laboratoire utilisé pour notre étude ne pouvant assurer une agitation adéquate, nous avons utilisé pour toutes les expériences un volume de $100 \mu \mathrm{l}$ par chambre après avoir vérifié que l'équilibre gazeux s'établissait très rapidement au travers de la faible épaisseur de liquide.

L'unité microrespirométrique est assemblée, puis immergée, hormis le réservoir $(R)$ dans le bain thermostaté à $30{ }^{\circ} \mathrm{C}$; cette température choisie pour toutes les expériences, correspond à celle utilisée pour la méthode de référence.

Lorsque l'équilibre thermique et hygrométrique est atteint (environ 30 min après l'immersion), le liquide manovolumétrique est déposé dans le réservoir (fig 1B). II pénètre par capillarité et gravité dans le capillaire. La colonne ainsi formée atteint une longueur d'équilibre $L_{0}$ qui dépend du volume d'air résiduel dans la chambre d'incubation et des propriétés physiques du liquide employé. La longueur $L_{0}$ est mesurée pour chaque unité respirométrique. Elle permet de calculer leur sensibilité propre (autocalibration). Le respiromètre est ensuite isolé de l'extérieur et le système de régulation de pression est mis en service. Les imprécisions de régulation de pression et de température à l'instant $t$ sont évaluées par la mesure des variations de la longueur de la colonne de liquide $L_{4}$ de l'unité témoin supposée stabilisée.

Le principe de la microrespirométrie à pression et volume variables a déjà été décrit par Verdier (1983); toutefois nous pouvons rappeler que la mesure de l'allongement de la colonne de liquide (dl) (fig 1C), survenu pendant l'intervalle de temps écoulé entre l'instant t et $t+d t$ permet de calculer les variations simultanées de pression $(\mathrm{d} P$ ) et de volume $(\mathrm{d} V)$ à l'intérieur de la chambre selon les relations suivantes :

$$
d V=-s \cdot d / \quad d P=\rho \cdot d l
$$

avec $\rho=$ densité du liquide manovolumétrique, $s=$ section du capillaire.

Reportées dans la forme différentielle de la loi d'Etat des gaz parfaits : $\mathrm{d} N R T=P \cdot \mathrm{d} V+$ $V \cdot d P$, ces variations de pression et de volume permettent de calculer les diminutions de la quantité gazeuse et ainsi d'évaluer la consommation d'oxygène à l'instant $t: \mathrm{O}_{2(t)}$. Elle s'exprime en nanolitre d'oxygène par microlitre de lait et par heure $(\mathrm{nl} / \mu \mathrm{l} / \mathrm{h})$ ramenée aux conditions standard de température $(273 \mathrm{~K})$ et de pression (10336 mm $\mathrm{H}_{2} \mathrm{O}$ ). Dans les conditions expérimentales de notre étude, un millimètre d'allongement de la colonne de liquide équivaut à une consommation de l'ordre de $10 \mathrm{nl}$ d'oxygène.

Les mesures d'allongement ont été faites visuellement à des intervalles de temps variant entre 3 et $10 \mathrm{~min}$ car adaptés à l'activité respiratoire de chaque échantillon. L'analyse respirométrique était stoppée lorsque la colonne de liquide manovolumétrique occupait toute la lumière du tube capillaire ou que le nombre de mesures effectuées en phase exponentielle de consommation d'oxygène était jugé suffisant.

\section{Inhibiteurs de croissance bactérienne}

Deux antibiotiques ont été utilisés simultanément pour inhiber la croissance de la plupart des espèces bactériennes du lait cru : la pénicilline $\mathrm{G}$ (100 UI/ml de lait) et l'acide nalidixique (40 mg/l de lait).

\section{Analyse statistique}

La justesse des mesures de consommation d'oxygène a été calculée, conformément aux recommandations de la norme FIL 128 (Anonyme, 1985); avant les calculs statistiques, les nombres d'UFC/ml ainsi que les consommations $\mathrm{d}^{\prime} \mathrm{O}_{2}$ ont subi une transiormation logarithmique pour tenter de normaliser les distributions.

La justesse représente l'étroitesse de l'accord entre la valeur vraie de la grandeur à mesurer et le résultat moyen qui serait obtenu en appliquant la technique expérimentale un grand nombre de fois. Par convention, la valeur vraie est donnée par le nombre UFC/ml obtenu par les techniques classiques de dénombrement (dans notre cas, moyenne de deux déterminations).

La justesse dépend de deux notions : l'exactitude de calibrage et la précision d'estimation. 


\section{Exactitude de calibrage}

Conformément à la norme FIL, l'équation de calibrage $(y=b \bullet x+a)$ a été établie selon la méthode des moindres carrés à partir des moyennes des deux déterminations faites sur chaque échantillon pour les deux techniques; les logarithmes décimaux des nombres d'UFC/ $\mathrm{ml}$ sont considérés comme variable dépendante et les logarithmes décimaux des consommations d'oxygène comme variable indépendante, pour donner une estimation de l'écart type résiduel dans l'unité de la méthode de référence. Nous avons appliqué la norme FIL établie pour les méthodes chimiques bien que certaines hypothèses ne soient pas vérifiées dans le cas des techniques microbiologiques : en particulier, le calcul du coefficient de régression et de l'écart type résiduel suppose une erreur négligeable dans la mesure des $x$ et des $y$, ce qui n'est pas le cas pour la plupart de ces méthodes. Pour que la méthode soit exactement calibrée, il faut que le coefficient de régression b de l'équation ne soit pas statistiquement différent de 1,000 .

\section{Précision d'estimation}

La précision d'estimation de la technique expérimentale est donnée par les limites de l'intervalle de confiance à l'intérieur duquel se situe, avec une probabilité de $95 \%$, la vraie donnée par la méthode de référence. Si le nombre d'UFC/ml y est calculé à partir de la droite de calibrage, ces limites sont en valeurs logarithmiques, log $y \pm$ $1,96 \cdot s_{y, x}$ où $s_{y, x}$ est l'écart-type résiduel des écarts à la droite de régression. En données non transformées, ces limites sont $y / 10^{1,96 \cdot s}$ pour la limite inférieure et y $\times 10^{1,96 \cdot s_{y \times x}}$ pour la litmite supérieure.

\section{RÉSULTATS}

Contribution des cellules somatiques à l'activité respiratoire initiale du lait

La figure 2A présente un exemple de l'évolution de l'activité respiratoire obtenue lors d'une incubation à $30^{\circ} \mathrm{C}$ d'un échantillon de lait prélevé aseptiquement à la mamelle ( 2 jet) et d'un échantillon prélevé dans le pot trayeur immédiatement après la traite (lait pot trayeur).

Au début de l'incubation, les échantillons de lait présentent des activités respiratoires voisines qui évoluent ensuite différemment : celle du lait $2^{e}$ jet décroît légèrement puis se stabilise autour de $1 \mathrm{nl} / \mu \mathrm{l} / \mathrm{h}$, tandis que celle du lait pot trayeur augmente de façon exponentielle à partir de la $2^{e}$ heure d'incubation pour atteindre après $4 \mathrm{~h}$, des niveaux d'activité 10 fois plus importants que celle du lait trait aseptiquement.

La figure $2 \mathrm{~B}$ présente les cinétiques de consommation d'oxygène de l'échantillon de lait prélevé dans le pot trayeur obtenues avec ou sans addition de deux inhibiteurs de la croissance bactérienne. L'activité respiratoire du lait contenant les inhibiteurs est initialement inférieure à celle du lait non traité; elle décroît ensuite légèrement pour se stabiliser autour de 1 $\mathrm{nl} / \mathrm{\mu l} / \mathrm{h}$ après 1 à $2 \mathrm{~h}$ d'incubation. Cette évolution est parallèle à celle du lait de traite aseptique $2^{e}$ jet présentée sur la figure $2 \mathrm{~A}$; les consommations d'oxygène de ces 2 échantillons aux différents temps d'incubation ne sont d'ailleurs pas significativement différentes à $P=0,05$ (test $t$ par paires; Dagnelie, 1970). De même, il n'existe pas de différence significative à $P=0,05$ entre les consommations d'oxygène du lait $2^{e}$ jet additionné ou non des inhibiteurs.

Ces résultats confirment que l'activité respiratoire somatique est non négligeable pour des laits fraîchement traits et qu'elle participe en grande partie à la phase plateau qui peut être assimilée à un bruit de fond. 


\section{Mesure de la consommation d'oxygène bactérienne}

Si l'on soustrait point par point les valeurs de consommation d'oxygène du lait $2^{e}$ jet ou du lait récolté en pot trayeur additionné des antibiotiques de celles mesurées sur l'échantillon pot trayeur, on obtient deux nouvelles courbes qui sont présentées sur la figure $2 \mathrm{C}$. Avec des coefficients de cor-

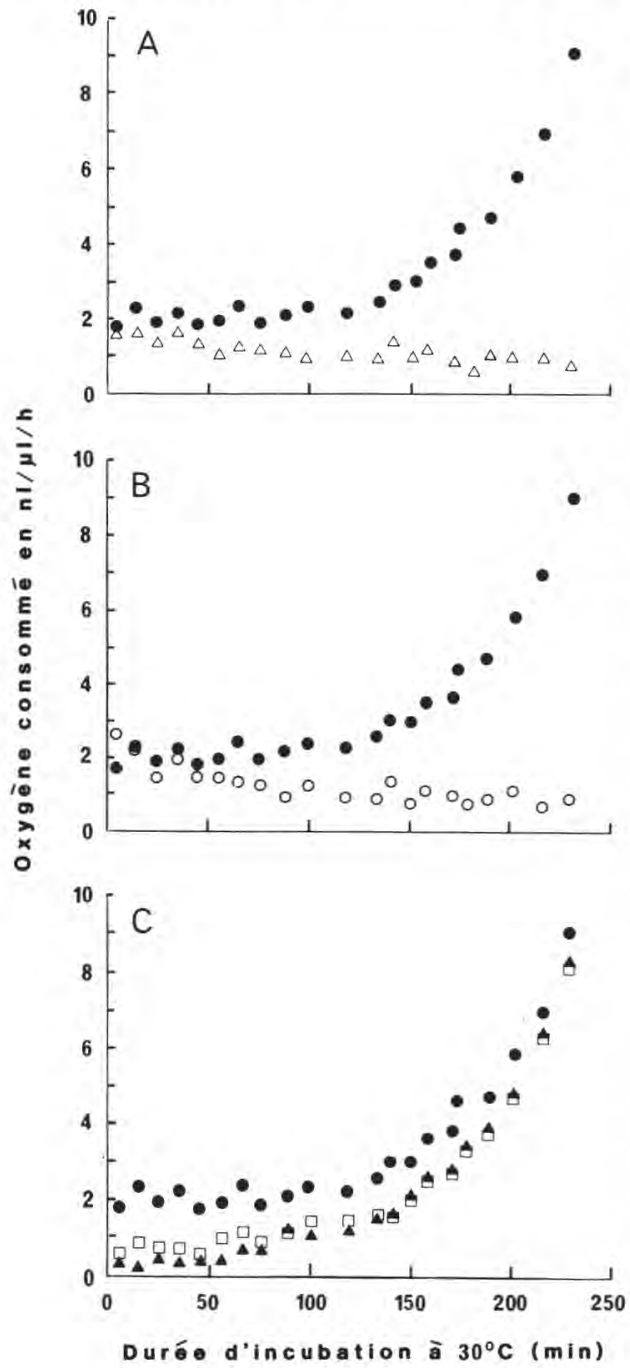

rélation respectifs de 0,93 et 0,95 , ces courbes s'ajustent de façon très étroite à une courbe de croissance exponentielle d'équation :

$$
N=N_{0} \cdot 2^{(t-t)} / T
$$

avec $N=$ nombre de bactéries au temps $t$; $N_{0}$ : nombre de bactéries au temps $t_{0} ; T=$ temps de génération.

Fig 2. Contribution respective des cellules somatiques et des bactéries à l'activité respiratoire du lait : A - évolution de la consommation d'oxygène mesurée sur 2 échantillons de lait analysés aussitôt après la traite; $B$ - effet des inhibiteurs de croissance bactérienne (Pénicilline G et Acide nalidixique) sur l'activité respiratoire du lait prélevé dans le pot trayeur; C - évolution de la consommation d'oxygène d'origine bactérienne estimée pour l'échantillon prélevé dans le pot trayeur.

(๑) échantillon prélevé dans le pot trayeur; $(\Delta)$ échantillon prélevé aseptiquement à la mamelle; $2 e$ jet; $(O)$ échantillon prélevé dans le pot trayeur et additionné de pénicilline $\mathrm{G}$ et d'acide nalidixique; ( $\square$ ) différence entre les valeurs de consommation d'oxygène de l'échantillon pot trayeur et de l'échantillon $2 e$ jet prélevé aseptiquement; ( $\mathbf{A})$ différence entre les valeurs de consommation d'oxygène de l'échantillon pot trayeur et le même échantillon additionné des inhibiteurs.

Contribution of somatic cells and bacteria to the respiratory activity: $A$ - evolution of oxygen consumption measured on 2 milk samples analysed immediately after milking; $B$ - effect of bacterial growth inhibitors (penicillin and nalidixic acid) on the respiratory activity of a milk sample taken in a milking pail; $C$ - evolution of bacterial oxygen consumption estimated on a milk sample taken in a milking pail.

(Ө) sample taken from a milking pail; ( $\Delta$ ) milk sample aseptically drawn from individual quarter; second stream; $(O)$ sample taken from a milking pail after the addition of penicillin $G$ and nalidixic acid; (ㅁ) difference between oxygen consumption values of the sample taken from a milking pail and the second stream aseptically drawn sample; (A) difference between the oxygen consumption values of the sample taken from a milking pail and the same sample mixed with inhibitors. 
En revanche, cet ajustement est nettement moins satisfaisant pour l'échantillon pot trayeur $(r=0,90)$. Cependant, après $4 \mathrm{~h}$ d'incubation environ, ces 3 courbes se rejoignent et les consommations d' $\mathrm{d}_{2}$ suivent la même croissance exponentielle; ainsi, nous avons émis l'hypothèse que toute mesure effectuée pendant cette phase de croissance peut être attribuée à l'activité respiratoire des différentes espèces bactériennes aérobies présentes. En effet, celle-ci a alors atteint un niveau suffisamment élevé pour rendre le bruit de fond somatique comparativement négligeable.

Relation entre la consommation d'oxygène et le nombre de bactéries du lait après une préincubation des échantillons

La figure 3 présente la relation existant entre les logarithmes décimaux de la con-

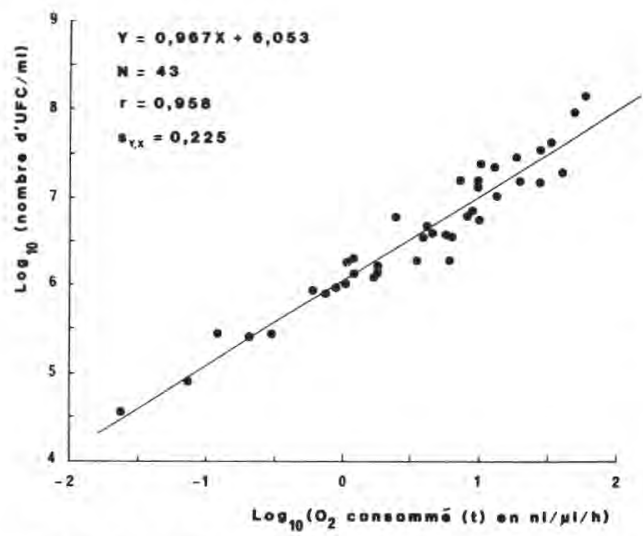

Fig 3. Relation entre les consommations $d^{\prime} \mathrm{O}_{2}$ et les nombres d'UFC/ml (en valeurs logarithmiques) mesurés après $4 \mathrm{~h}$ d'incubation $\left(30^{\circ} \mathrm{C}\right)$ des échantillons de lait cru.

Relationship between the oxygen consumption and the number of CFU/ml (in $\log _{10}$ ) determined after $4 \mathrm{~h}$ of incubation $\left(30^{\circ} \mathrm{C}\right)$ of raw milk samples. sommation d'oxygène et du nombre UFC/ $\mathrm{ml}$, déterminés simultanément sur 43 échantillons incubés pendant $4 \mathrm{~h}$ minimum à $30^{\circ} \mathrm{C}$. Tous prélevés dans une ferme de la région de Brunoy, une partie de ces échantillons fut préalablement conservée à basse température pendant des durées variables, de façon à couvrir une gamme de concentration variant de $5 \times 10^{4}$ à $5 \times$ $10^{7} \mathrm{UFC} / \mathrm{ml}$. L'équation de la droite de régression linéaire est la suivante :

$\log _{10}(\mathrm{UFC} / \mathrm{ml})=0,967 \cdot \log _{10}\left(\mathrm{O}_{2(\mathrm{t})}\right)+6,053$

avec un écart type résiduel $\left(s_{y, x}\right)$ de 0,225 et un coefficient de corrélation linéaire $(r)$ de 0,958 . Avec une erreur standard $\left(s_{b}\right)$ de 0,045 , le coefficient de régression $b=$ $0,967 \pm 0,088$ n'est pas statistiquement différent de 1,000 à $P=0,95$.

\section{Prédiction de la consommation d'oxy- gène bactérienne initiale}

L'étude précédente a mis en évidence l'étroitesse de la relation entre une mesure de consommation d'oxygène et un nombre de bactéries. Cependant, au temps zéro de l'incubation $\left(t_{0}\right)$, la mesure d'une consommation d'oxygène ne permet pas l'estimation de l'activité respiratoire des bactéries, compte tenu de l'importance du bruit de fond somatique enregistré sur des laits crus. L'expérience a montré qu'après un temps d'incubation suffisant (environ $4 \mathrm{~h}$ pour la majorité des échantillons), la consommation d'oxygène suit une courbe de croissance exponentielle. Ainsi, il est possible de prédire l'activité respiratoire initiale au temps $\left(t_{0}\right)$ par extrapolation de la droite obtenue lors de la représentation semi-logarithmique des données (fig 4), et de calculer le temps de doublement moyen de la consommation $\mathrm{d}^{\prime} \mathrm{O}_{2}$ à partir de son coefficient de régression. 


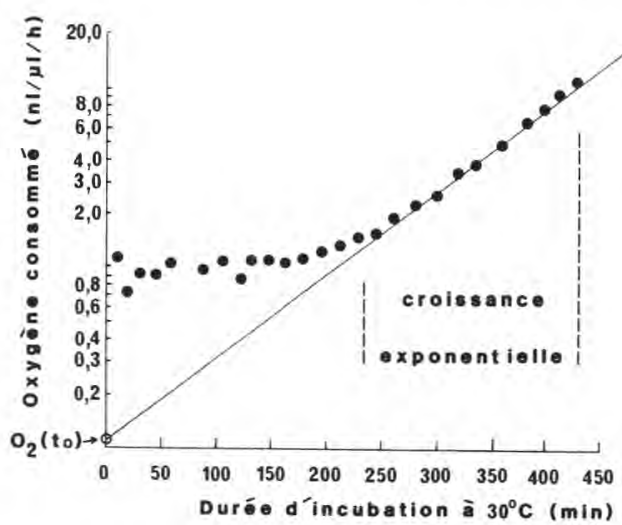

Fig 4. Mode de prédiction de l'activité respiratoire bactérienne initiale d'un échantillon de lait. Method of prediction of the initial bacterial respiratory activity of a milk sample.

Un programme de calcul a été établi sur ordinateur, pour éviter le travail fastidieux et peu précis d'une interprétation graphique. Par des régressions successives, il recherche la portion de courbe qui s'ajuste avec le meilleur coefficient de corrélation à une courbe de croissance exponentielle, et ensuite, il calcule l'ordonnée à l'origine et le coefficient de régression de la droite obtenue en coordonnées semi-logarithmiques.

\section{Relation entre la consommation d'oxy- gène bactérienne initiale prédite et le nombre initial d'UFC/mI}

\section{Précision d'estimation}

La droite de régression présentée par la figure 5 a été établie à partir des logarithmes décimaux des moyennes de deux déterminations réalisées pour chaque échantillon : d'une part, le nombre initial d'UFC/ml et d'autre part, la consommation d'oxygène bactérienne initiale prédite. L'équation de la droite calculée sur 94 échantillons couvrant des gammes de concentration de $6 \times 10^{3}$ à $10^{7} \mathrm{UFC} / \mathrm{ml}$ est la suivante:

$$
\begin{aligned}
\log _{10}(\mathrm{UFC} / \mathrm{ml}) & =1,028 \cdot \log _{10}\left(\mathrm{O}_{2(\mathrm{to})}\right) \\
+ & 5,938
\end{aligned}
$$

avec un écart type résiduel $\left(s_{y, x}\right)$ de 0,177 et un coefficient de corrélation linéaire $(r)$ de 0,973 .

La précision d'estimation de la technique microrespirométrique est donc de \pm $0,177 \times 1,96= \pm 0,346 \log _{10}$ UFC/ml. Si le nombre d'UFC/ml est estimé à partir de la droite de régression, les limites de l'intervalle de confiance de y (à $P=0,95$ ) sont en données non transformées :

$$
\begin{gathered}
y / 10^{0,346} \text { et } y \times 10^{0,346}, c^{\prime} e s t-a ̀-d i r e, \\
y \times 0,451 \text { et } y \times 2,219
\end{gathered}
$$

\section{Exactitude de calibrage}

Avec une erreur standard $\left(s_{b}\right)$ de 0,020 , le coefficient de régression $b=1,028 \pm 0,039$ n'est pas statistiquement différent de 1,000. De plus, l'ordonnée à l'origine de la droite de régression linéaire ne diffère pas significativement de celle obtenue pour les données déterminées après une incubation des échantillons pendant $4 \mathrm{~h}$ (fig 3 ).

La technique étant correctement calibrée (pente de 1), l'ordonnée à l'origine nous permet d'estimer de façon simple le nombre initial d'UFC/ml $(y)$ à partir de la consommation d'oxygène initiale prédite par extrapolation ( $x$ en $\mathrm{nl} / \mathrm{\mu l} / \mathrm{h}$ ) :

$$
\begin{gathered}
\log _{10} y=\log _{10} x+5,958, \text { d'où, } \\
y=x \times 10^{5,958}=x \times 9,08 \times 10^{5} \mathrm{UFC} / \mathrm{ml}
\end{gathered}
$$




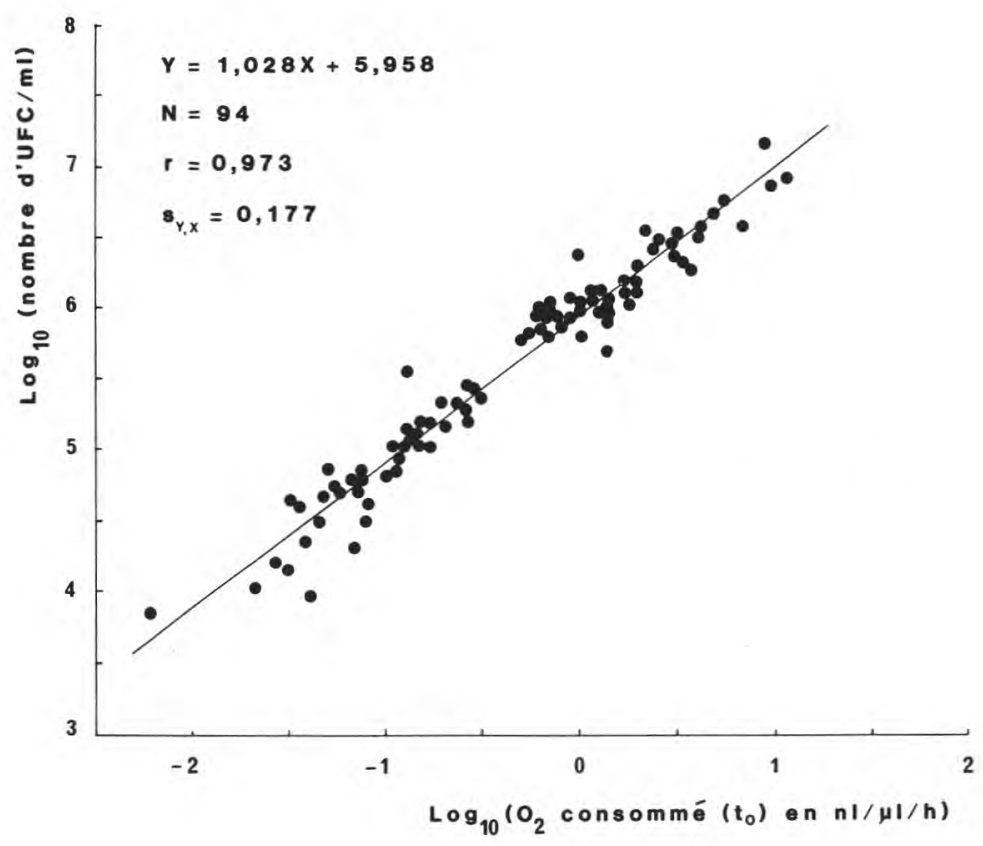

Fig 5. Relation entre les consommations bactériennes d'oxygène initiales prédites $\left(\mathrm{O}_{2(\mathrm{to})}\right)$ et les nombres réels d'UFC/ml (en valeurs logarithmiques) des échantillons de lait à cet instant.

Relationship between the predicted bacterial initial oxygen consumptions $\left(\mathrm{O}_{2(t o)}\right)$ and the actual numbers of CFU/ml (in $\log _{10}$ ) in raw milk samples at that time.

Temps de doublement moyen de la consommation d'oxygène

Sur l'ensemble des échantillons analysés, couvrant une période de l'année de janvier à juin, ce temps de doublement moyen est de $60 \mathrm{~min}$ avec un écart type de $14 \mathrm{~min}$.

\section{DISCUSSION ET CONCLUSIONS}

Cette étude nous a permis de valider l'utilisation des valeurs de consommations d'oxygène mesurées par microrespirométrie pour évaluer la qualité bactériologique du lait cru. Dans un premier temps, nous avons mis en évidence la nécessité de préincuber les échantillons avant toute mesure, compte tenu du rôle important que joue la respiration des cellules somatiques sur la valeur de consommation d'oxygène initiale mesurée sur du lait cru. En effet, les échantillons contenant des niveaux très faibles de micro-organismes ou pour lesquels la croissance bactérienne est bloquée par addition d'inhibiteurs, présentent une activité respiratoire non négligeable qui décroît ensuite au cours de l'incubation à $30^{\circ} \mathrm{C}$, probablement en raison de la diminution physiologique de la vitalité des cellules somatiques après la traite (Luck et al, 1970b). En revanche, après quelques heures de préincubation à 
$30^{\circ} \mathrm{C}$, la respiration bactérienne supplante celle des cellules somatiques, en raison de la croissance microbienne intense à cette température. Grâce à l'extrapolation à l'origine des valeurs mesurées en phase de croissance exponentielle, nous pouvons prédire la consommation $\mathrm{d}^{\prime} \mathrm{O}_{2}$ d'origine strictement bactérienne dans l'échantillon de lait avant incubation. Avec cet ajustement, nous avons donc émis deux hypothèses : premièrement, l'absence de phase de latence pour les micro-organismes, et deuxièmement, l'existence d'une consommation moyenne d' $\mathrm{O}_{2}$ constante par cellule bactérienne au cours de l'incubation. On peut penser que ces deux hypothèses sont vérifiées car la droite de calibrage obtenue entre les logarithmes du nombre initial de micro-organismes et de la consommation bactérienne initiale prédite ne diffère pas significativement de celle obtenue avec des résultats mesurés après $4 \mathrm{~h}$ d'incubation; en effet, l'existence d'une phase de latence ou de variations de consommation $d^{\prime} \mathrm{O}_{2}$ par cellule auraient conduit à des ordonnées à l'origine différentes. Enfin, il est généralement admis que la phase de latence de la plupart des espèces dominantes du lait cru est très courte à $30^{\circ} \mathrm{C}$.

Par ailleurs, la consommation d'oxygène moyenne par cellule bactérienne ne varie pas en fonction du niveau de contamination des échantillons puisque la pente de la droite de calibrage ne diffère pas significativement de 1,000. Ceci signifie en outre que la nature de la flore a peu d'incidence sur la respiration bactérienne car elle varie en fonction du niveau de contamination du lait. En effet, des échantillons peu contaminés contiennent généralement des microcoques et des streptocoques en flore dominante, tandis que la proportion de bactéries à Gram négatif devient très importante dans des laits pollués (Thomas et al, 1962; Twomey et Crawley, 1968; Richard, 1983). Enfin, les Pseudomonas constituent la flore dominante des laits conservés longtemps à basse température (Richard, 1981).

Contrairement aux consommations d'oxygène par UFC, les temps de doublement de cette consommation varient de façon assez importante d'un échantillon à l'autre, probablement en raison de la grande variété de la flore. Malgré les modifications probables de la nature de la flore au cours de l'incubation à $30^{\circ} \mathrm{C}$, nous avons assimilé ces temps de doublement à des temps de génération moyens de la flore présente. Ces temps de génération calculés $(60 \pm 14 \mathrm{~min}$ ) sont en moyenne assez proches de ceux trouvés dans la littérature, bien que les résultats rapportés proviennent le plus souvent de tests réalisés sur des cultures pures de microorganismes et/ou sur des milieux autres que le lait cru et/ou sous des taux d'oxygénation variables (Olsen et Jezeski, 1963; Lee et Collins, 1976; Langeveld et Cuperus, 1980). Or les conditions de culture et la nature des flores en présence influencent la vitesse de croissance moyenne des micro-organismes. En effet, les bactéries thermorésistantes ne se développent pas ou très peu dans le lait cru en raison de la présence d'inhibiteurs naturels (Thomas et al, 1950), tandis que la vitesse de croissance des Pseudomonas du groupe fluorescent est très rapide et influencée par le taux d'oxygénation du milieu (Olsen et Jeseski, 1963). De plus, l'existence d'interactions microbiennes et/ ou un mauvais état physiologique de certaines cellules présentes dans le lait peuvent affecter le temps de génération moyen de la flore.

Contrairement aux résultats rapportés pour les mesures d'oxygène dissous (Pickering et Jayne-Williams, 1963; Stadhouders, 1965; Hadland, 1966; Luck et al, 1968 et 1970a), la précision d'estimation calculée à partir des consommations d'oxy- 
gène estimées est très satisfaisante (environ $\left.0,350 \log _{10} \mathrm{UFC} / \mathrm{ml}\right)$. La différence observée se situe au niveau du principe même de la technique utilisée pour mesurer la consommation d'oxygène au sein de l'échantillon. La mesure de la chute du taux d'oxygène dissous dans l'échantillon de lait après une incubation de 3 à 4 heures à l'aide d'une électrode spécifique, ne tient pas compte de l'incidence de deux facteurs importants : d'une part, la contribution des cellules somatiques (Luck et al, $1970 \mathrm{~b})$, et d'autre part, le temps de doublement moyen de la consommation d'oxygène par la flore bactérienne. Or, grâce aux expériences réalisées avec le microrespiromètre, nous avons montré que ces deux facteurs peuvent entraîner au bout de 3 à 4 heures d'incubation des consommations totales d'oxygène variables d'un échantillon à l'autre, pour un même niveau initial de bactéries.

La justesse des consommations d'oxygène du lait estimées par microrespirométrie est donc sensiblement équivalente à celle des techniques directes, telle la Deft $\left(s_{y, x}=0,245 \log _{10}\right.$ UFC/ml d'après Dasen et al., 1987). De plus, elle est nettement plus satisfaisante que celle des autres techniques indirectes développées au cours des dernières années, comme le dosage de l'ATP par bioluminescence (Bossuyt, 1981; Buchet, 1985) ou l'impédancemétrie (Firstenberg-Eden et Tricarico, 1983; Suhren et Heeschen, 1987; Piton et Dasen, 1988). Cependant, des résultats récemment présentés (Eriksen et Olsen, 1988; Waes et al, 1989) permettent d'espérer une nette amélioration de la sensibilité et de la précision pour l'ATPmétrie.

Toute cette étude a été réalisée sur un microrespiromètre prototype de laboratoire, mais l'application de cette technique en routine nécessite une automatisation de l'appareil. Cette version non encore commercialisée, aura l'avantage d'être très simple d'utilisation. Une agitation magnétique au sein de chaque chambre permettra l'analyse d'un volume d'échantillon plus important entraînant l'abaissement de la limite de détection et du temps de réponse de la technique. De plus, un système de régulation de pression interne très sensible, ainsi que la lecture optique de l'allongement de la colonne de liquide vont contribuer encore à l'amélioration de la précision de la lecture. Une étude complémentaire sera alors nécessaire pour tester les caractéristiques analytiques de la technique automatisée, en particulier sa fidélité.

De nombreuses applications peuvent être envisagées pour la microrespirométrie dans le domaine laitier, comme par exemple, la numération de différents groupes de micro-organismes telle la flore psychrotrophe grâce à l'addition d'antibiotiques ou la sélection de températures plus faibles ou l'étude du métabolisme (homo ou hétérofermentaire) des bactéries lactiques. Des contrôles de stérilité sur une chaîne de fabrication sont également concevables, après une préincubation des échantillons.

\section{REMERCIEMENTS}

Nous remercions vivement $M$. R Grappin, directeur de la Station de Recherches en Technologie et Analyses Laitières (INRA, Poligny), pour ses suggestions données lors de la rédaction de ce manuscrit. Ce travail a bénéficié d'une aide financière de l'agence nationale de valorisation de la recherche (Contrat ANVAR-CNRS $n^{\circ}$ A 861049 Q).

\section{RÉFÉRENCES}

Anonyme (1972) Standard methods for the examination of dairy products. American Public Health Association, 13th edn.. New York 
Anonyme (1980) Microbial Ecology of Foods. Vol. 1. Factors Affecting Life and Death of Micro-organisms. International Commission on Microbiological Specifications for Foods. Academic Press, New York

Anonyme (1981) Lait liquide. Dénombrement des micro-organismes (technique par comptage des colonies à $30^{\circ} \mathrm{C}$ ). Norme Provisoire FIL-IDF 100

Anonyme (1985) Milk.Definition and evaluation of the overall accuracy of indirect methods of milk analysis. Application to calibration procedure and quality control in dairy laboratory. Norme provisoire FIL-IDF 128

Bossuyt R (1981) Determination of bacteriological quality of raw milk by an ATP assay technique. Milchwissenschaft 36, 257-260

Buchet C (1985) Appréciation rapide de la qualité bactériologique du lait grâce au dosage de I'ATP par bioluminescence. DEA Science et Technologie du Lait, Faculté des Sciences de Caen

Campbell JE, Gilchrist JE (1973) Spiral plate technique for counting bacteria in milk and other foods. Dev Ind Microbiol 14, 95-102

Dagnelie P (1970) Théorie et Méthodes statistiques. Vol. 2. Les Méthodes de l'Inférence statistique. Editions J. Duculot SA, Gembloux

Dasen A, Piton C, Grappin R, Guerry P (1987) Evaluation de la technique DEFT associée à un comptage visuel ou à un comptage par un analyseur d'images pour la numération de la flore totale du lait cru. Lait 67, 77-95

Donnelly CB, Gilchrist JE, Peeler JT, Campbell JE (1976) Spiral plate count method for the examination of raw and pasteurized milk. Appl Environ Microbiol 32, 21-27

Eriksen B, Olsen O (1988) Rapid assessment of the microbial status of bulk milk and raw meat with the new instrument Bactofoss. Presented at ATP 88-Symposium on Rapid Microbiology, University of Sussex, Brighton, U.K.

Firstenberg-Eden R, Tricarico M (1983) Impedimetric determination of total, mesophilic and psychrotrophic counts in raw milk. J Food Sci $48,1750-1754$

Gilchrist JE, Campbell JE, Donnely CB, Peeler JT, Delaney JM (1973) Spiral plate method for bacterial determination. Appl Microbiol 25, 244-252
Hadland G (1966) Oxygen measurements as a basis for classification of milk. Proc XVII Int Dairy Congr B, 233-237

Janvis B, Lach VH, Wood JM (1977) Evaluation of the spiral plate maker for the enumeration of micro-organisms in foods. J Appl Bacteriol $43,149-157$

Langeveld L, Cuperus $F(1980)$ The relation between temperature and growth rate in pasteurized milk of different types of bacteria which are important to the determination of that milk. Neth Milk Dairy J 34, 106-125

Lee DA, Collins EB (1976) Influences of temperature on growth of $S$. cremoris and $S$. lactis. J Dairy Sci 59, 405-409

Luck $H$ (1972) Bacteriological quality tests for bulk-cooled milk. Dairy Sci Abstr 34, 101-122

Luck H, Clark PC, Van Tonder JL (1970a) Oxygen content as a basis for classifying bulkcooled milk. Milchwissenschaft 25, 155-160

Luck H, Du Toit JJ, Hermann MN (1968) The oxygen content of milk as an index of its bacterial count. S Afr J Agric Sci 11, 141-152

Luck H, Giesecke WH, Du Toit JJ, Van Tonder $\mathrm{JL}$ (1970b) The influence of epithelial cells and of polymorphonuclear and mononuclear leucocytes on the methylene blue and resazurin tests and the oxygen content of milk. $S$ Afr J Dairy Technol 2, 241-251

Olsen R, Jezeski J (1963) Some effects of carbon source, aeration, and temperature on growth of a psychrotrophic strain of Pseudomonas fluorescens. J Bacteriol 86, 429-433

Peeler JT, Gilchrist JE, Donnelly CB, Campbell JE (1977) A collaborative study of the spiral plate method for examining milk samples. $J$ Food Prot 40, 462-464

Pickering A, Jayne-Williams DJ (1963) Preliminary observations on the measurement of dissolved oxygen in milk as an index of keeping quality. J Soc Dairy Technol 16, 28-30

Piton C, Dasen A (1988) Evaluation de la mesure d'impédance comme technique rapide d'appréciation de la qualité bactériologique du lait cru. Lait 68, 467-484

Richard J (1981) Influence de diverses méthodes de nettoyage des machines à traire sur la qualité de conservation du lait cru à basse température. Lait 61, 354-369 
Richard J (1983) Nature de la flore microbienne dominante et sous dominante des laits crus très pollués. Lait $63,148-170$

Richard J, Auclair J (1967) Etude des contaminations microbiennes à la production. Rev Lait Fr 242, 227-230

Stadhouders J (1965) The polarographic measurement of the dissolved oxygen in milk as an index of the bacteriological quality of milk. Neth Milk Dairy J 19, 119-138

Suhren G, Heeschen W (1987) Impedance assays and the bacteriological testing of milk and milk products. Milchwissenschaft 42, 619-627

Thomas SB, Egdell JW, Clegg LFL, Cuthbert WA (1950) Thermoduric organisms in milk. Part I. A review of the literature. Proc Soc Appl Bacteriol 13, 27-64

Thomas SB, Hobson PM, Bird ER, King KP, Drucer G, Cox DR (1962) The microflora of raw milk as determined by plating on yeastrel-milk agar incubated at $30^{\circ} \mathrm{C}$. J Appl Bacteriol 25, 107-115

Twomey A, Crawley WE (1968) The microflora of raw milk. NZJ Dairy Technol 3, 44-48

Verdier B (1983) Le respiromètre à pression et volume variables. Une technique simple et sensible pour l'étude écophysiologique des animaux du sol. In: New Trends in Soil Biology. Proc. VIII Int. Colloquium of Soil Zoology. Louvain-La-Neuve, Belgique, August 30September 1983 (Lebrun P, André HM, De Medts A, Gregoire Wibo C, Wauthy G, eds) Ottignies-Louvain-La-Neuve, pp 369-386

Waes G, Van Crombrugge J, Reybroeck W (1989) The ATP-F test for estimation of bacteriological quality of raw milk. International Seminar on Modern Microbiological Methods, Santander, May 1989 